\title{
Generalization of pinching operation to binary matroids
}

\author{
Vahid Ghorbani, Ghodratollah Azadi, Habib Azanchiler
}

\begin{abstract}
In this paper, we generalize the pinching operation on two edges of graphs to binary matroids and investigate some of its basic properties. For $n \geq 2$, the matroid that is obtained from an $n$-connected matroid by this operation is a $k$-connected matroid with $k \in\{2,3,4\}$ or is a disconnected matroid. We find conditions to guarantee this $k$. Moreover, we show that Eulerian binary matroids are characterized by this operation and we also provide some interesting applications of this operation.
\end{abstract}

2010 MSC: 05B35

Keywords: Binary matroid, Connectivity, Pinching, Splitting, Splitting off, Element splitting

\section{Introduction}

The matroid and graph notations and terminology used here will follow [4] and [7]. Let $G$ be a graph and $S$ be a subset of the edge set $E(G)$. We denote by $G_{S}^{p}$ the graph that is obtained from $G$ by adding a new vertex $w$ and replacing any edge $u v \in S$ with two edges $u w$ and $w v$. The transition from $G$ to $G_{S}^{p}$ is called the $k$-pinching operation [2] where $k=|S|$. If $S$ is the empty set, then 0-pinching means adding a new single vertex $w$. If $|S|=1$, then applying the pinching operation is equivalent to the subdivision of $u v$ where $S=\{u v\}$. If $S=E(G)$, then we have the parallel classes on the new vertex $w$ on $G_{S}^{p}$ such that the sequence of the cardinality of them is equal to the degree sequence of the vertices of $G$.

Applying the pinching operation on graphs is a useful method for solving $2 k$-edge-connectivity problems for graphs [2]. For example, suppose that $G$ is a $2 k$-edge-connected graph. Then the graph $G^{\prime}$ obtained from $G$ by pinching together any $k$ edges of $G$ is also 2 k-edge-connected.

Raghunathan, Shikare, and Wapare [5] extended the splitting operation and Azadi [1] extended the splitting off and the element splitting operations from graphs to binary matroids. These operations are defined as follows.

Definition 1.1. Let $M$ be a binary matroid on a set $E$ and $A$ be a matrix that represents $M$ over $G F(2)$. Consider two elements $x$ and $y$ of $E(M)$. Let $A_{x, y}$ be the matrix that is obtained by adjoining an extra

Vahid Ghorbani (Corresponding Author), Ghodratollah Azadi, Habib Azanchiler; Department of Mathematics, UrmiaUniversity, Iran (email:v.ghorbani@urmia.ac.ir,gh.azadi@urmia.ac.ir,h.azanchiler@urmia.ac.ir) 
row to $A$ whose entries are zero everywhere except in the columns corresponding to $x$ and $y$ where it takes value 1. Let $M_{x, y}$ be the matroid that represents by the matrix $A_{x, y}$. Then the transition from $M$ to $M_{x, y}$ is called the splitting operation.

Definition 1.2. Let $M$ be a binary matroid on a set $E$ and $A$ be a matrix that represents $M$ over $G F(2)$. Consider two elements $x$ and $y$ of $E(M)$. Let $A_{x, y}^{\prime}$ be the matrix that is obtained by adjoining an extra row to $A$ whose entries are zero everywhere except in the columns corresponding to $x$ and $y$ where it takes value 1 and then adjoining an extra column to the resulting matrix with this column being zero everywhere except in the last row. Let $M_{x, y}^{\prime}$ be the matroid that represents by the matrix $A_{x, y}^{\prime}$. Then the transition from $M$ to $M_{x, y}^{\prime}$ is called the element splitting operation.

Definition 1.3. Let $M$ be a binary matroid on a set $E$ and $A$ be a matrix that represents $M$ over $G F(2)$. Consider two elements $x$ and $y$ of $E(M)$. Let $A_{x y}$ be the matrix that is obtained by adjoining an extra column to $A$ which is the sum of the columns corresponding to $x$ and $y$, and then deleting the two columns corresponding to $x$ and $y$. Let $M_{x y}$ be the matroid that represents by the matrix $A_{x y}$. Then the transition from $M$ to $M_{x y}$ is called the splitting off operation.

Splitting off operation on graphs is defined as follows.

Definition 1.4. Let $G$ be a graph. Consider two adjacent non-loop edges $x=u w$ and $y=v w$. The splitting off a pair $(x, y)$ of edges from a vertex $w$ means that we replace the edges $x$ and $y$ by a new edge $\alpha=u v$. If $u=v$ then the resulting loop is deleted from the graph.

\section{2-pinching operation in 2-connected graphs}

Let $G$ be a 2-connected graph with the edge set $E(G)$ and $S \subseteq E(G)$ where $S=\left\{x=u_{1} v_{1}, y=u_{2} v_{2}\right\}$. To apply the 2-pinching operation, we first delete $x$ and $y$ and add new vertex $w$. Then we add new edges $x^{\prime}=u_{1} w, x^{\prime \prime}=w v_{1}, y^{\prime}=u_{2} w$ and $y^{\prime \prime}=w v_{2}$. When $x$ and $y$ are adjacent, we take $v_{1}=v_{2}=v$. Therefore, $x^{\prime}=u_{1} w, x^{\prime \prime}=w v, y^{\prime}=u_{2} w$ and $y^{\prime \prime}=w v$ (Figure 1). We denote by $G_{x y}^{p}$ the graph that is obtained by applying the 2-pinching operation on edges $x$ and $y$. Note that we can retrieve the graph $G$ from $G_{x y}^{p}$ by splitting off the pair $\left(x^{\prime}, x^{\prime \prime}\right)$ and then splitting off the pair $\left(y^{\prime}, y^{\prime \prime}\right)$.

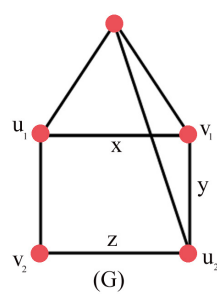

(G)



$\left(\mathrm{G}_{\mathrm{xz}}^{\mathrm{p}}\right)$

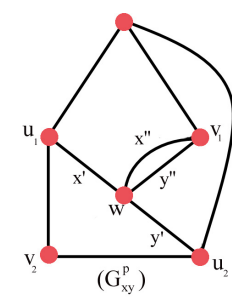

$\left(\mathrm{G}_{\mathrm{xy}}^{\mathrm{p}}\right)$

Figure 1. 2-pinching operation on $\{x, z\}$ and $\{x, y\}$.

Now let $G$ be a 2-connected graph and $x, y \in E(G)$. Then $G$ has a cycle $C_{n}$ of length $n$ containing both $x$ and $y$. Assume that the cycle $C_{n}$ has the minimum cardinality among all such cycles. After applying the 2-pinching operation on $x$ and $y$, the cycle $C_{n}$ transforms into two cycles $C_{k+2}^{\prime}$ and $C_{n-k}^{\prime}$ where $k$ is the length of shortest path $Q$ among endpoints of $x$ and $y$ such that $x^{\prime \prime}, y^{\prime \prime} \in C_{k+2}^{\prime}$ and $x^{\prime}, y^{\prime} \in$ $C_{n-k}^{\prime}$. In other words, $C_{k+2}^{\prime}=E(Q) \cup\left\{x^{\prime \prime}, y^{\prime \prime}\right\}^{1}$ and $C_{n-k}^{\prime}=\left(C_{n}-(E(Q) \cup\{x, y\})\right) \cup\left\{x^{\prime}, y^{\prime}\right\}$. Note that if $k=0$, then $x$ and $y$ are adjacent and $C_{n}$ transforms to $C_{2}^{\prime}=\left\{x^{\prime \prime}, y^{\prime \prime}\right\}$ and $C_{n}^{\prime}=\left(C_{n}-\{x, y\}\right) \cup\left\{x^{\prime}, y^{\prime}\right\}$.

${ }^{1}$ In this section, for cycle $C_{n}$ of length $n$ from a graph $G$, we consider just its edge set 
Theorem 2.1. Let $G$ be a graph and $x, y \in E(G)$. Suppose that $G$ has a cycle $C_{n}$ with a minimum length containing both $x$ and $y$. Let $x^{\prime}, x^{\prime \prime}, y^{\prime}, y^{\prime \prime}, C_{k+2}^{\prime}$ and $C_{n-k}^{\prime}$ be the notations as mentioned above. Moreover, let $\delta=\left\{C_{k+2}^{\prime}, C_{n-k}^{\prime}\right\}$ and $C$ be a subset of edges of $G_{x y}^{p}$. Then $C$ forms a cycle of $G_{x y}^{p}$ if and only if $C \in \delta$ or $C$ satisfies one of the following conditions.

(i) $C$ is a cycle of $G$ which contains neither $x$ nor $y$;

(ii) $C=\left(C^{\prime}-\{x\}\right) \cup\left\{x^{\prime}, x^{\prime \prime}\right\}$ where $C^{\prime}$ is a cycle of $G$ containing $x$ but not $y$, or $C=\left(C^{\prime \prime}-\{y\}\right) \cup\left\{y^{\prime}, y^{\prime \prime}\right\}$ where $C^{\prime \prime}$ is a cycle of $G$ containing $y$ but not $x$; and

(iii) $C=C_{1} \Delta C_{2}$ where $C_{1} \in \delta$, and $C_{2}$ is a cycle of type (i) or (ii) such that $C_{1} \cap C_{2} \neq \emptyset$.

Proof. It is clear that if $C \in \delta$ or if $C$ is a cycle of $G$ containing neither $x$ nor $y$, then $C$ is a cycle of $G_{x y}^{p}$. Now suppose that $C=\left(C^{\prime}-\{x\}\right) \cup\left\{x^{\prime}, x^{\prime \prime}\right\}=\left(C^{\prime}-\left\{u_{1} v_{1}\right\}\right) \cup\left\{u_{1} w, v_{1} w\right\}$ where $C^{\prime}$ is a cycle of $G$ containing $x$ but not $y$ and has length $k^{\prime}+1$, so

$$
C^{\prime}=v_{1} x u_{1} e_{1} z_{1} \ldots e_{k^{\prime}-1}^{\prime} z_{k^{\prime}-1} e_{k^{\prime}} v_{1} .
$$

After applying the pinching operation on $x$ we delete $x$ and add $x^{\prime}=u_{1} w$ and $x^{\prime \prime}=v_{1} w$. Thus

$$
\left(C^{\prime}-\{x\}\right) \cup\left\{x^{\prime}, x^{\prime \prime}\right\}=u_{1} e_{1} z_{1} \ldots e_{k^{\prime}-1} z_{k^{\prime}-1} e_{k^{\prime}} v_{1} x^{\prime \prime} w x^{\prime} u_{1} .
$$

Therefore $C$ is a cycle of $G_{x y}^{p}$. Similarly, if $C=\left(C^{\prime \prime}-\{y\}\right) \cup\left\{y^{\prime}, y^{\prime \prime}\right\}=\left(C^{\prime}-\left\{u_{2} v_{2}\right\}\right) \cup\left\{u_{2} w, v_{2} w\right\}$ where $C^{\prime \prime}$ is a cycle of $G$ containing $y$ but not $x$, then $C$ is a cycle of $G_{x y}^{p}$. Now suppose that $C_{1}$ is a cycle of type (i) or (ii) and $C_{2} \in \delta$ such that $C_{1} \cap C_{2} \neq \emptyset$. To show that $C_{1} \Delta C_{2}$ is a cycle of $G_{x y}^{p}$, we consider the following two possible cases.

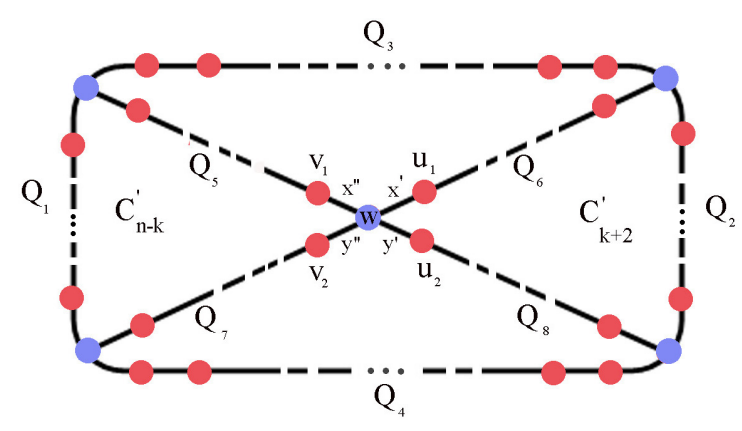

Figure 2. Internally disjoint paths.

Case(1) Let $C_{1}$ be a cycle of type (i). Assume that $C_{1}$ has a non-empty intersection with both $C_{k+2}^{\prime}$ and $C_{n-k}^{\prime}$. Let $Q_{1}$ and $Q_{2}$ be the paths such that $C_{1} \cap C_{n-k}^{\prime}=E\left(Q_{1}\right)$ and $C_{1} \cap C_{k+2}^{\prime}=E\left(Q_{2}\right)$. Moreover, let $Q_{3}, Q_{4}$ be internally disjoint paths which have the same end vertices as of $Q_{1}$ and $Q_{2}$ such that $C_{1}=E\left(Q_{1}\right) \cup E\left(Q_{2}\right) \cup E\left(Q_{3}\right) \cup E\left(Q_{4}\right)$ (See figure 2). Suppose that $C_{n-k}^{\prime}=E\left(Q_{1}\right) \cup E\left(Q_{5}\right) \cup E\left(Q_{7}\right)$ and $C_{k+2}^{\prime}=E\left(Q_{2}\right) \cup E\left(Q_{6}\right) \cup E\left(Q_{8}\right)$ where $Q_{i}$ and $Q_{j}$ for $i, j \in\{1,2,5,6,7,8\}$ are internally disjoint paths. Then clearly $C_{1} \Delta C_{n-k}^{\prime}$ and $C_{1} \Delta C_{k+2}^{\prime}$ are cycles of $G_{x y}^{p}$. Similarly, if $C_{1}$ has a non-empty intersection with exactly one of $C_{k+2}^{\prime}$ or $C_{n-k}^{\prime}$, then $C_{1} \Delta C_{n-k}^{\prime}$ or $C_{1} \Delta C_{k+2}^{\prime}$ is a cycle of $G_{x y}^{p}$.

Case(2) Let $C_{2}=E\left(Q_{3}\right) \cup E\left(Q_{5}\right) \cup E\left(Q_{6}\right)$ and $C_{3}=E\left(Q_{4}\right) \cup E\left(Q_{7}\right) \cup E\left(Q_{8}\right)$. Clearly, $C_{2}$ and $C_{3}$ are cycles of type (ii). It is easy to see that $C_{2} \Delta C_{n-k}^{\prime}, C_{2} \Delta C_{k+2}^{\prime}, C_{3} \Delta C_{n-k}^{\prime}$ and $C_{3} \Delta C_{k+2}^{\prime}$ are cycles of $G_{x y}^{p}$.

Now suppose that $x$ and $y$ are adjacent. Then $v_{1}=v_{2}=v$ and $C_{k+2}^{\prime}=v y^{\prime \prime} w x^{\prime \prime} v$. An argument similar to one as given above shows that $C_{1} \Delta C_{2}^{\prime}$ and $C_{1} \Delta C_{n}^{\prime}$ are cycles of $G_{x y}^{p}$ where $C_{1}$ is a cycle of type (i) or (ii) such that $C_{1} \cap C_{n}^{\prime} \neq \emptyset$. 
Conversely, let $C$ be a cycle of $G_{x y}^{p}$. By the definition of $G_{x y}^{p}$ one can see that $x, y \notin E\left(G_{x y}^{p}\right)$ and $x^{\prime}, x^{\prime \prime}, y^{\prime}, y^{\prime \prime} \notin E(G)$. Let $N=\left\{x^{\prime}, x^{\prime \prime}, y^{\prime}, y^{\prime \prime}\right\}$. We know that the induced subgraph of $N$ is either the graph (a) or the graph (b) of the following figure.

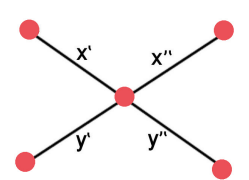

(a)

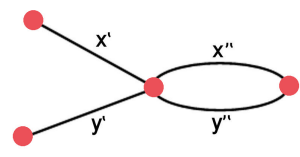

(b)

Figure 3. Two possible induced subgraphs of $N$.

It is straightforward to check that if $C$ is a cycle of $G_{x y}^{p}$, then $C$ contains a subset $N^{\prime}$ of $N$ with $\left|N^{\prime}\right| \in\{0,2\}$. Therefore, every cycle of $G_{x y}^{p}$ is a cycle of type (i), (ii) or (iii).

\section{2-pinching operation for binary matroids}

Now, by using Theorem 2.1 we extend the notion of the 2-pinching operation from 2-connected graphs to binary matroids.

Let $M$ be a binary matroid and $x, y \in E(M)$. We denote by $\mathcal{C}_{p}$ the collection of all the circuits of $M$ which contain both $x$ and $y$. Choose a member of $\mathcal{C}_{p}$ and denote it by $C_{x y}$. Partition $C_{x y}-\{x, y\}$ into sets $X_{1}$ and $X_{2}$ where $X_{1}$ or $X_{2}$ may be empty. We denote such a partition by $P=\left(X_{1}, X_{2}\right)$. Clearly, when $\mathcal{C}_{p}$ is empty or $\left|C_{x y}\right|=2$, there is no such partition and we say that $P$ is empty.

Definition 3.1. Let $A$ be a matrix that represents the matroid $M$ over $G F(2)$. For $x, y \in E(M)$, let $\mathcal{C}_{p} \neq \emptyset$ and $P=\left(X_{1}, X_{2}\right)$ constructed as above. Let $A_{x y}^{P}$ be the matrix obtained from $A$ by the following way.

(1) Adjoin four extra columns to $A$ with the labels $\alpha_{1}, \alpha_{2}, \alpha_{3}$ and $\alpha_{4}$ such that:

- The corresponding entries of $\alpha_{1}$ and $x$ are equal;

$-\alpha_{2}$ is a zero column;

- $\alpha_{3}, \alpha_{4}$ are columns whose entries are 1 or 0 such that sum of the corresponding entries of the corresponding columns of $X_{1} \cup\left\{\alpha_{1}, \alpha_{3}\right\}$ and $X_{2} \cup\left\{\alpha_{2}, \alpha_{4}\right\}$ are zero.

(2) Delete the two columns corresponding to $x$ and $y$

(3) Adjoin an extra row with this row being zero everywhere except in the columns corresponding to the new elements $\alpha_{k}$ where it takes value 1 , for $1 \leq k \leq 4$.

We call a vector matroid obtained in this way a pinching on $x$ and $y$ with respect to $P$ or 2-pinching with respect to $P$ and denoted it by $M_{x y}^{P}$.

Definition 3.2. Let all hypotheses of Definition 3.1 be established except that $\mathcal{C}_{p}{ }^{2}$ is an empty set. To build $A_{x y}^{\emptyset}$ from $A$, carry out all the steps of Definition 3.1 with the following changes.

- The corresponding entries of $\alpha_{1}$ and $x$ are equal;

- The corresponding entries of $\alpha_{3}$ and $y$ are equal;

2 This means $x$ or $y$ or both are cocircuits of $M$ 
- $\alpha_{2}$ and $\alpha_{4}$ are zero columns;

Remark 3.3. Let $x$ and $y$ be two elements of a matroid $M$. Note that, when $\left|C_{x y}\right|=2$, we can build $A_{x y}^{\emptyset}$ with both Definition 3.1 and Definition 3.2. So throughout this paper, we may assume that $\mathcal{C}_{p} \neq \emptyset$ unless in special cases of some proofs.

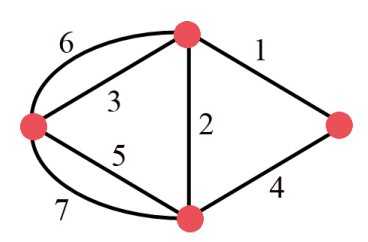

Figure 4. $\quad\{x=6, y=7\}$ and $P=(\{1\},\{4\})$.

Example 3.4. Consider the cycle matroid of the graph in Figure 4 with the following matrix $A$ over GF(2) that represents it.

$$
A=\left[\begin{array}{lllllll}
1 & 2 & 3 & 4 & 5 & 6 & 7 \\
1 & 0 & 0 & 1 & 0 & 0 & 0 \\
0 & 1 & 0 & 1 & 1 & 0 & 1 \\
0 & 0 & 1 & 0 & 1 & 1 & 1
\end{array}\right]
$$

Let $x=6$ and $y=7$. Then $\mathcal{C}_{p}=\{\{2,6,7\},\{1,4,6,7\}\}$. Let $C_{x y}=\{1,4,6,7\}$. Partition $C_{x y}-\{x, y\}$ to two sets $X_{1}=\{1\}$ and $X_{2}=\{4\}$. So by definition 3.1, after applying the pinching operation on $x$ and $y$ with respect to $P_{1}=(\{1\},\{4\})$, the matrix $A$ transforms into the following matrix.

$$
A_{x y}^{P_{1}}=\left[\begin{array}{ccccccccc}
1 & 2 & 3 & 4 & 5 & \alpha_{1} & \alpha_{2} & \alpha_{3} & \alpha_{4} \\
1 & 0 & 0 & 1 & 0 & 0 & 0 & 1 & 1 \\
0 & 1 & 0 & 1 & 1 & 0 & 0 & 0 & 1 \\
0 & 0 & 1 & 0 & 1 & 1 & 0 & 1 & 0 \\
0 & 0 & 0 & 0 & 0 & 1 & 1 & 1 & 1
\end{array}\right]
$$

One can easily check that the vector matroid of $A_{x y}^{P_{1}}$ is isomorphic to $M^{*}\left(K_{3,3}\right)$. Hence the 2-pinching operation on a graphic matroid may not yield a graphic matroid. Moreover, $M^{*}\left(K_{3,3}\right)$ is a 3-connected matroid while the original matroid is not. Clearly, if $C_{x y}=\{2,6,7\}$, then $P_{2}=(\{2\}, \emptyset)$ and $M_{x y}^{P_{2}}$ is a graphic matroid.

Theorem 3.5. Let $M=(E, \mathcal{C})$ be a binary matroid on the set $E$ together with the collection $\mathcal{C}$ of circuits. Let $x, y \in E(M)$, and for a given circuit $C_{x y}$, let $P=\left(X_{1}, X_{2}\right)$ be a partition of $C_{x y}-\{x, y\}$. Suppose that $\Gamma=\left\{\alpha_{1}, \alpha_{2}, \alpha_{3}, \alpha_{4}\right\}$ such that $\alpha_{i} \notin E(M)$ for $1 \leq i \leq 4$. Then $M_{x y}^{P}=\left((E-\{x, y\}) \cup \Gamma, \mathcal{C}_{x y}^{P}\right)$ with $\mathcal{C}_{x y}^{P}=\mathcal{C}_{0} \cup \mathcal{C}_{1} \cup \delta \cup \mathcal{C}_{2} \cup \mathcal{C}_{3}$ is a binary matroid where

(i) $\mathcal{C}_{0}=\{C \in \mathcal{C}: C$ contains neither $x$ nor $y\}$;

(ii) $\mathcal{C}_{1}=\left\{(C-\{x\}) \cup\left\{\alpha_{1}, \alpha_{2}\right\}: C \in \mathcal{C}\right.$, and $C$ contains $x$ but not $\left.y\right\} \cup\left\{(C-\{y\}) \cup\left\{\alpha_{3}, \alpha_{4}\right\}: C \in \mathcal{C}\right.$, and $C$ contains $y$ but not $x\}$;

(iii) $\delta=\left\{X_{1} \cup\left\{\alpha_{1}, \alpha_{3}\right\}, X_{2} \cup\left\{\alpha_{2}, \alpha_{4}\right\}\right\}$;

(iv) $\mathcal{C}_{2}=\left\{C_{1} \Delta C_{2}: C_{1} \in \mathcal{C}_{0} \cup \mathcal{C}_{1}\right.$ and $C_{2} \in \delta$, and if $C_{1} \in \mathcal{C}_{0}$, then $C_{1} \cap C_{2} \neq \emptyset$; and there is no circuit $C$ of $\mathcal{C}_{0}$ and $\mathcal{C}_{1}$ such that $\left.C \subseteq C_{1} \Delta C_{2}\right\}$; and 
(v) $\mathcal{C}_{3}=$ The set of minimal members of $\left\{(C-\{x, y\}) \cup \Gamma: x, y \in C\right.$ and $\left.C \neq C_{x y}\right\}$.

Proof. It is easy to see that every member of $\mathcal{C}_{x y}^{P}$ is a circuit in $M_{x y}^{p}$. To show that $\mathcal{C}_{x y}^{p}$ is the collection of circuits of a binary matroid on $(E-\{x, y\} \cup \Gamma)$, we must show that if $X, Y \in \mathcal{C}_{x y}^{P}$, then $X \nsubseteq Y$ and $\mathrm{Y} \nsubseteq \mathrm{X}$, and $X \Delta Y$ contains at least one member of $\mathcal{C}_{x y}^{P}$. So there are fifteen cases to check and checking them is straightforward.

Let $A$ be a matrix that represents $M$ over $G F(2)$. Let $C \subseteq E(M)$. Then $C$ is a circuit of $M$ if and only if $C$ is minimal and the mod-2 sum of the columns of $A$ corresponding to $C$ is a zero columns [4]. By using this fact, a consequence of the last theorem is the following partial result.

Corollary 3.6. Under the hypotheses of Theorem 3.5, Let A be a matrix that represents $M$ over $G F(2)$, then the collection of minimal dependent sets of vector matroid of the matrix $A_{x y}^{P}$ is $\mathcal{C}_{x y}^{P}$.

Proof. Depending on how to construct the matrix $A_{x y}^{P}$, the proof is straightforward.

Corollary 3.7. Under the hypotheses of Theorem 3.5, Let $r(M)$ and $r\left(M_{x y}^{P}\right)$ be the ranks of $M$ and $M_{x y}^{P}$, respectively. Then $r\left(M_{x y}^{P}\right)=r(M)+1$.

Proof. Suppose that $B$ is a basis of $M$ such that $C_{x y}$ is a fundamental circuit with respect to $B$. Then exactly one of the following holds.

(i) $\left(C_{x y}-\{x\}\right) \subseteq B$.

Let $B_{1}=(B-\{y\}) \cup\left\{\alpha_{3}, \alpha_{4}\right\}$. Then $\left|B_{1}\right|=|B|+1$. By Theorem 3.5(iii), $C_{1}^{\prime}=X_{1} \cup\left\{\alpha_{1}, \alpha_{3}\right\}$ and $C_{2}^{\prime}=X_{2} \cup\left\{\alpha_{2}, \alpha_{4}\right\}$ are circuits of $M_{x y}^{P}$. Clearly, $B_{1}$ does not contain either $C_{1}^{\prime}$ or $C_{2}^{\prime}$, nor any member of $\mathcal{C}_{0} \cup \mathcal{C}_{1} \cup \mathcal{C}_{2} \cup \mathcal{C}_{3}$. So $B_{1}$ is an independent set. Since $B_{1} \cup \alpha_{1}$ contains $C_{1}^{\prime}$ and $B_{1} \cup \alpha_{2}$ contains $C_{2}^{\prime}$, the set $B_{1}$ is a maximal independent set and so $B_{1}$ is a basis of $M_{x y}^{P}$.

(ii) $\left(C_{x y}-\{y\}\right) \subseteq B$.

Similar to (i) we can see that if $B_{2}=(B-\{x\}) \cup\left\{\alpha_{1}, \alpha_{2}\right\}$, then $B_{2}$ is a maximal independent set and $\left|B_{2}\right|=|B|+1$.

(iii) $C_{x y}-\{z\} \subseteq B$ where $z \notin\{x, y\}$.

Suppose that $z \in X_{1}$ and $B_{3}=(B-\{x, y\}) \cup\left\{\alpha_{1}, \alpha_{2}, \alpha_{3}, \alpha_{4}\right\}$. Consider two sets $\left(X_{1}-\{z\}\right) \cup\left\{\alpha_{1}, \alpha_{3}\right\}$ and $X_{2} \cup\left\{\alpha_{2}, \alpha_{4}\right\}$. Clearly, $\left(X_{1}-\{z\}\right) \cup\left\{\alpha_{1}, \alpha_{3}\right\}$ is an independent set and $X_{2} \cup\left\{\alpha_{2}, \alpha_{4}\right\}$ is a circuit of $M_{x y}^{P}$. Hence $B_{3}$ cannot be a basis of $M_{x y}^{P}$, but $B_{4}=B_{3}-\left\{z^{\prime}\right\}$ where $z^{\prime} \in\left(X_{2} \cup\left\{\alpha_{2}, \alpha_{4}\right\}\right)$ is a basis of $M_{x y}^{P}$ and $\left|B_{4}\right|=|B|+1$.

By (i),(ii) and (iii), we conclude that $r\left(M_{x y}^{P}\right)=r(M)+1$.

\section{Connectivity of $M_{x y}^{P}$}

In this section, by studying the cardinality of some circuits and cocircuits of $M_{x y}^{P}$, we explore the relationship between connectivity of a given connected binary matroid $M$ and $M_{x y}^{P}$.

Theorem 4.1. Let $M_{x y}^{P}$ be the pinching of a connected binary matroid $M$. If $\mathcal{C}_{1} \cup \mathcal{C}_{3}=\emptyset$, then $M_{x y}^{P}$ is a disconnected matroid. Otherwise, $M_{x y}^{P}$ is a connected matroid. 
Proof. Since $M$ is a connected matroid, for every pair $(x, y)$ of two elements of $E(M)$, there exists a circuit of $\mathcal{C}(M)$ containing both $x$ and $y$. Now assume $\mathcal{C}_{1} \cup \mathcal{C}_{3}=\emptyset$. By Theorem 3.5, we conclude that there is no circuit of $M$ which contains $x$ but not $y$, also there is no circuit of $M$ which contains $y$ but not $x$. Let $P=\left(X_{1}, X_{2}\right)$. Then $\delta=\left\{C_{1}^{\prime}=X_{1} \cup\left\{\alpha_{1}, \alpha_{3}\right\}, C_{2}^{\prime}=X_{2} \cup\left\{\alpha_{2}, \alpha_{4}\right\}\right\}$ and $\mathcal{C}_{2}=\left\{C_{1}^{\prime} \Delta C: C \in\right.$ $\mathcal{C}_{0}$, and $\left.C \cap C_{1}^{\prime} \neq \emptyset\right\} \cup\left\{C_{2}^{\prime} \Delta C: C \in \mathcal{C}_{0}\right.$, and $\left.C \cap C_{2}^{\prime} \neq \emptyset\right\}$. Hence every member of $\mathcal{C}_{2}$ contains either $\left\{\alpha_{1}, \alpha_{3}\right\}$ or $\left\{\alpha_{2}, \alpha_{4}\right\}$. Since $\mathcal{C}_{x y}^{p}=\mathcal{C}_{0} \cup \delta \cup \mathcal{C}_{2}$, there is no member of $\mathcal{C}_{x y}^{P}$ containing $\left\{\alpha_{1}, \alpha_{2}\right\},\left\{\alpha_{3}, \alpha_{4}\right\}$, $\left\{\alpha_{1}, \alpha_{4}\right\}$ and $\left\{\alpha_{2}, \alpha_{3}\right\}$. Therefore $M_{x y}^{P}$ is a disconnected matroid. Similarly, it can be shown that if $\mathcal{C}_{1} \cup \mathcal{C}_{3}$ is non-empty, then $M_{x y}^{P}$ is a connected matroid.

Definition 4.2. We say that two elements $x$ and $y$ from given matroid $M$ are equivalent and denoted by $x \sim y$, if at least one of $x$ and $y$ is a coloop of $M$ or $\{x, y\}$ is a cocircuit of $M$.

If a matroid $M$ has at least one pair of equivalent elements, then $M$ is a connected or is a disconnected matroid. Our important task of this section is to rely connectivity of obtained matroid by applying the 2pinching operation on a given $n$-connected matroid. So we investigate the cocircuits of matroid obtained by this operation.

It is well known that all circuits and all cocircuits of an $n$-connected matroid with at least $2(n-1)$ elements have at least $n$ elements [4]. For a given binary matroid $M$ with matrix representation $A$ and two elements $x, y \in E(M)$, we denote by $\mathcal{R}\left(C^{*}\right)$ and $\mathcal{R}\left(C_{p}^{*}\right)$ the cocircuit spaces of $A$ and $A_{x y}^{P}$, respectively. In the following lemmas, we use the fact that the intersection of a circuit and a cocircuit of a binary matroid $M$ has even cardinality [4].

Lemma 4.3. Let $M_{x y}^{P}$ be a pinching of a binary matroid $M$. Then $\Gamma=E\left(M_{x y}^{P}\right)-E(M)$ is a cocircuit of $M_{x y}^{p}$ if and only if $x$ is not equivalent to $y$.

Proof. Suppose that $M_{x y}^{P}$ is such a pinching with $P=\left(X_{1}, X_{2}\right)$ and $\Gamma=\left\{\alpha_{1}, \alpha_{2}\right.$,

$\left.\alpha_{3}, \alpha_{4}\right\}$. Let $A$ be the matrix that represent $M$ over $G F(2)$. Build $A_{x y}^{P}$ for $M_{x y}^{P}$ according to Definition 3.1. Since there is a row in the row space of $A_{x y}^{P}$ which this row is zero everywhere except in the corresponding entries of $\Gamma$, we get $\Gamma \in \mathcal{R}\left(C_{p}^{*}\right)$. Assume, without loss of generality, that $x \sim y$. Then exactly one of the following holds.

(i) $\{x, y\}$ is a cocircuit of $M$. Then, there is a row in the row space of $A$ such that has the following form

$$
\begin{aligned}
& E(M)-\{x, y\} \quad x \quad y \\
& {\left[\begin{array}{llll|ll}
0 & 0 & \ldots & 0 & 1 & 1
\end{array}\right] .}
\end{aligned}
$$

Let $\delta_{X_{i}}$ be the sum of the corresponding entries of $X_{i}$ in this row, for $i \in\{1,2\}$. Clearly, $\delta_{X_{1}}=$ $\delta_{X_{2}}=0$. Thus, after applying the pinching operation on $M$, we have the following row in the row space of $A_{x y}^{P}$

$$
\begin{gathered}
E(M)-\{x, y\} \\
{\left[\begin{array}{cccc|cccc}
0 & 0 & \ldots & 0 & 1 & 0 & 1 & 0
\end{array}\right] .}
\end{gathered}
$$

We conclude that $\left\{\alpha_{1}, \alpha_{3}\right\}$ and $\left\{\alpha_{2}, \alpha_{4}\right\}$ are in $\mathcal{R}\left(C_{p}^{*}\right)$. By Theorem 3.5, $C_{1}=X_{1} \cup\left\{\alpha_{1}, \alpha_{3}\right\}$ and $C_{2}=X_{2} \cup\left\{\alpha_{2}, \alpha_{4}\right\}$ are circuits of $M_{x y}^{P}$ and since $\left|C_{j} \cap\left\{\alpha_{i}\right\}\right|=1$ for some $j \in\{1,2\}$ and some $i \in\{1,2,3,4\}$. Hence $\left\{\alpha_{1}, \alpha_{3}\right\}$ and $\left\{\alpha_{2}, \alpha_{4}\right\}$ are minimal and so $\Gamma$ is not a cocircuit of $M_{x y}^{P}$.

(ii) $\{x\}$ is a cocircuit of $M$. Then $M$ is a disconnected matroid and so $\left\{\alpha_{2}, \alpha_{4}\right\}$ is a circuit of $M_{x y}^{\emptyset}$. We see that in the row space of $A_{x y}^{\emptyset}$ the corresponding entries of $\alpha_{1}$ and $\alpha_{3}$ are 1 and 0 , respectively and $\delta_{X_{1}}=\delta_{X_{2}}=0$. Therefore $\left\{\alpha_{1}\right\}$ is a cocircuit of $M_{x y}^{P}$ and so $\Gamma$ is not. 
To complete the proof, we must show that $\Gamma$ is minimal when $x$ is not equivalent to $y$. On the contrary, let $Z$ be a proper subset of $\Gamma$ which is minimal. Clearly, if $|Z|=1$ or 3 , then there is a circuit in $M_{x y}^{P}$ such that its intersection with $Z$ or $Z \Delta \Gamma$ is a singleton set and if $|Z|=2$, we get $x \sim y$; a contradiction. Hence $\Gamma$ is a cocircuit of $M_{x y}^{P}$.

For a given matroid $M$, The girth $g(M)$ is the minimum circuit size of $M$ unless $M$ has no circuits, in which case, $g(M)=\infty$. The cogirth is the girth of the dual of $M$. By Lemma 4.3, we conclude that if $M$ is an $n$-connected matroid with $n \geq 4$, then the cogirth of $M_{x y}^{P}$ is 4 . Now suppose that the girth of such a matroid $M$ is $k$ and $\left|C_{x y}\right|=k$. Then clearly, $k \geq n$ and by Theorem 3.5, there is a matroid $M_{x y}^{P}$ which will decrease the girth of $M$ to $w$ where $2 \leq w \leq\left[\frac{k}{2}\right]+1$. Note that if $g(M)=\infty$, for a given matroid $M$, then $g\left(M_{x y}^{\emptyset}\right)=2$.

Lemma 4.4. Let $M$ be an $n$-connected binary matroid with $n \geq 4$ and $|E(M)| \geq 2(n-1)$. Let $x, y \in$ $E(M)$ and $P=\left(X_{1}, X_{2}\right)$ be a partition of $C_{x y}-\{x, y\}$ with $X_{i} \neq \emptyset$ for $i \in\{1,2\}$. Then $\left(\Gamma, E\left(M_{x y}^{P}\right)-\Gamma\right)$ is a 4-separation of $M_{x y}^{P}$ where $\Gamma=E\left(M_{x y}^{P}\right)-E(M)$.

Proof. Suppose that $M$ is an $n$-connected binary matroid with $n \geq 4$ and $|E(M)| \geq 2(n-1)$. Let $M_{x y}^{P}$ be a pinching of $M$ with respect to partition $P$ with non-empty parts. Then $\left|E\left(M_{x y}^{P}\right)\right| \geq 2 n$. Consider the partition $(X, Y)$ of $E\left(M_{x y}^{P}\right)$ with $X=\Gamma$ and $Y=E\left(M_{x y}^{p}\right)-\Gamma$ where $\Gamma=E(M)-E\left(M_{x y}^{P}\right)$. Clearly, $\min \{|X|,|Y|\}=4$. Suppose that $r$ and $r^{\prime}$ be the rank functions of $M$ and $M_{x y}^{P}$, respectively. The set $\Gamma$ is an independent set and $Y \subset E(M)$. By using Theorem 3.7

$$
r^{\prime}(X)+r^{\prime}(Y)-r^{\prime}\left(M_{x y}^{p}\right) \leq 4+r(M)-r^{\prime}\left(M_{x y}^{p}\right) \leq 3
$$

Hence, for $n \geq 4$, the partition $(X, Y)$ is a 4-separation of $M_{x y}^{P}$.

The last result says that for every $n$-connected matroid with $n \geq 4$, the maroid $M_{x y}^{P}$ is a $k$-connected matroid for some $k$ in $\{2,3,4\}$.

Lemma 4.5. Let $x$ and $y$ be two elements of an $n$-connected binary matroid $M$, for $n \geq 3$, and let $M_{x y}^{P}$ be a pinching of $M$. Let $Z$ be a cocircuit of $M_{x y}^{P}$ such that $Z \neq \Gamma$ where $\Gamma=E\left(M_{x y}^{P}\right)-E(M)$. Then $|Z| \geq n$.

Proof. Assume, without loss of generality, that $Z^{\prime}$ is an $n$-element cocircut of $M$ and $P=\left(X_{1}, X_{2}\right)$ is a partition of $C_{x y}-\{x, y\}$. Let $A$ be a matrix that represents $M$ and $A_{x y}^{P}$ constructed from $A$ by Definition 3.1. Suppose that $\delta_{X_{i}}$, for $i \in\{1,2\}$ is the sum of the corresponding entries of $X_{i}$ in the corresponding row of $Z^{\prime}$ in row space of $A$. So $\delta_{X_{i}} \in\{0,1\}$. Then exactly one of the following holds.

(i) $x, y \notin Z^{\prime}$ and $\left|Z^{\prime} \cap X_{1}\right|$ and $\left|Z^{\prime} \cap X_{2}\right|$ are even. Then $\delta_{X_{1}}=\delta_{X_{2}}=0$ and therefore all corresponding entries of $\Gamma$ in the corresponding row in row space of $A_{x y}^{P}$ are 0 . We conclude that $Z^{\prime}$ is a cocircuit of $M_{x y}^{P}$.

(ii) $x, y \notin Z^{\prime}$ and $\left|Z^{\prime} \cap X_{1}\right|$ and $\left|Z^{\prime} \cap X_{2}\right|$ are odd. Then $\delta_{X_{1}}=\delta_{X_{2}}=1$ and therefore the corresponding entries of $\alpha_{1}$ and $\alpha_{2}$ in $A_{x y}^{P}$ are 0 and $\alpha_{3}$ and $\alpha_{4}$ are 1 . So $Z^{\prime} \cup\left\{\alpha_{3}, \alpha_{4}\right\}$ is a cocircuit of $M_{x y}^{P}$.

Note that there is no cocircuit $Z^{\prime}$ of $M$ not containing $x$ and $y$ such that $\left|Z^{\prime} \cap X_{1}\right|$ is odd and $\left|Z^{\prime} \cap X_{2}\right|$ is even or vice versa. Since in this case $\left|Z^{\prime} \cap C_{x y}\right|$ is odd; a contradiction

(iii) $x$ is in $Z^{\prime}$ but $y$ not, then $\left|Z^{\prime} \cap X_{1}\right|$ is odd and $\left|Z^{\prime} \cap X_{2}\right|$ is even or vice versa. Then $\left(Z^{\prime}-\{x\}\right) \cup\left\{\alpha_{1}\right\}$ or $\left(Z^{\prime}-\{x\}\right) \cup\left\{\alpha_{1}, \alpha_{3}, \alpha_{4}\right\}$ is a cocircuit of $M_{x y}^{P}$.

(iv) $y$ is in $Z^{\prime}$ but $x$ not, then $\left|Z^{\prime} \cap X_{1}\right|$ is odd and $\left|Z^{\prime} \cap X_{2}\right|$ is even or vice versa. Then $\left(Z^{\prime}-\{y\}\right) \cup\left\{\alpha_{3}\right\}$ or $\left(Z^{\prime}-\{y\}\right) \cup\left\{\alpha_{4}\right\}$ is a cocircuit of $M_{x y}^{P}$. 
(v) $x, y \in Z^{\prime}$ and $\left|Z^{\prime} \cap X_{1}\right|$ and $\left|Z^{\prime} \cap X_{2}\right|$ are even. Then $\delta_{X_{1}}=\delta_{X_{2}}=0$ and therefore the corresponding entries of $\alpha_{2}$ and $\alpha_{4}$ in $A_{x y}^{P}$ are 0 and $\alpha_{1}$ and $\alpha_{3}$ are 1 . So $\left(Z^{\prime}-\{x, y\}\right) \cup\left\{\alpha_{1}, \alpha_{3}\right\}$ is a cocircuit of $M_{x y}^{P}$.

(vi) $x, y \in Z^{\prime}$ and $\left|Z^{\prime} \cap X_{1}\right|$ and $\left|Z^{\prime} \cap X_{2}\right|$ are odd. Then $\delta_{X_{1}}=\delta_{X_{2}}=1$ and therefore the corresponding entries of $\alpha_{2}$ and $\alpha_{3}$ in $A_{x y}^{P}$ are 0 and $\alpha_{1}$ and $\alpha_{4}$ are 1. So $\left(Z^{\prime}-\{x, y\}\right) \cup\left\{\alpha_{1}, \alpha_{4}\right\}$ is a cocircuit of $M_{x y}^{P}$.

Clearly, every cocircuit $Z$ in (i)-(vi) has at least $n$ elements and $x$ is not equivalent to $y$. By Lemma $4.3 \Gamma$ is a cocircuit of $M_{x y}^{P}$, and if $Z^{\prime \prime} \Delta \Gamma$ is a cocircuit of $M_{x y}^{P}$, then $\left|Z^{\prime \prime}\right| \geq n$.

Consider the following proposition [4], which is straightforward consequence of the fact that all circuits and all cocircuits of an $n$-connected matroid have at least $n$ elements where the ground set of such matroid has at least $2(n-1)$ elements.

Proposition 4.6. Let $(X, Y)$ be an n-separation of an n-connected matroid and suppose that $|X|=n$. Then $X$ is either a coindependent circuit or an independent cocircuit.

By using this Proposition and the last three lemmas, for a given $n$-connected matroid with $n \geq 4$, we have the following result to determine connectivity of pinching matroid with respect to $P$.

Theorem 4.7. Let $M$ be an $n$-connected binary matroid with $n \geq 4$ and $|E(M)| \geq 2(n-1)$. Then $M_{x, y}^{P}$ with respect to $P=\left(X_{1}, X_{2}\right)$ is a $k$-connected matroid for $k \in\{2,3,4\}$, if $\min \left\{\left|X_{1}\right|,\left|X_{2}\right|\right\} \geq k-2$.

Proof. Let $M$ be an $n$-connected binary matroid and let $C_{x y}$ be an $n^{\prime}$-element circuit of $M$ where $n^{\prime} \geq n$. Assume that $P=\left(X_{1}, X_{2}\right)$ with $\left|X_{1}\right|=n^{\prime}-k$ and $\left|X_{2}\right|=k-2$. Let $X=\left(X_{2} \cup\left\{\alpha_{2}, \alpha_{4}\right\}\right)$ and $Y=E\left(M_{x y}^{P}\right)-X$ and let $r$ and $r^{\prime}$ be the rank functions of $M$ and $M_{x y}^{P}$, respectively. Since $X$ is a circuit of $M_{x y}^{P}$, so $r^{\prime}(X)=k-1$. Clearly, $r^{\prime}(Y) \leq r^{\prime}\left(E\left(M_{x y}^{P}\right)\right.$. Now by these facts, we have

$$
r^{\prime}(X)+r^{\prime}(Y)-r^{\prime}\left(M_{x y}^{P}\right) \leq k-1 .
$$

Since $\min \{|X|,|Y|\}=k$, the partition $(X, Y)$ is a $k$-separation of $M_{x y}^{P}$. To complete the proof, we shall show that, $M_{x y}^{P}$ has no $j$-separation for $j<k$. On the contrary, let $\left(X^{\prime}, Y^{\prime}\right)$ be a $j$-separation of $M_{x y}^{P}$ with $\left|X^{\prime}\right|=j$. Then, by Proposition 4.6, the set $X^{\prime}$ must be a coindependent circuit or independent cocircuit in $M_{x y}^{P}$, But such circuit or cocircuit by Theorem 3.5 and Lemma 4.5 does not exist; a contradiction.

Definition 4.8. Let $M$ be an $n$-connected binary matroid and $x, y \in E(M)$ such that $x$ is not equivalent to $y$. We say that a collection of some subsets of $E\left(M_{x y}^{P}\right)$ is a Pinched set if all its members have at least $(n+1)$ elements. Note that if we consider $\mathcal{C}^{*}\left(M_{x y}^{P}\right)-\Gamma$ where $\Gamma=E\left(M_{x y}^{P}\right)-E(M)$, then the cardinality of its members can be characterized by Lemma 4.5.

Theorem 4.9. Let $M$ be an $n$-connected binary matroid for $n \in\{2,3\}$ and $x, y \in E(M)$ such that $x$ is not equivalent to $y$ and $\mathcal{C}_{p}$ has at least one member with $k$-elements where $k \geq 2 n$ and every $n$ element circuit of $M$ contains exactly one of $x$ and $y$. Let $\mathcal{C}^{*}\left(M_{x y}^{P}\right)-\Gamma$ is a Pinched set. Then $M_{x y}^{P}$ is an $(n+1)$-connected matroid.

Proof. Suppose that $M$ is an $n$-connected binary matroid and $x, y \in E(M)$. Choose $C_{x y}$ from $\mathcal{C}_{p}$ with $\left|C_{x y}\right| \geq 2 n$. Partition $C_{x y}-\{x, y\}$ to two sets $X_{1}$ and $X_{2}$ with $\left|X_{1}\right|=n-1$ and $\left|X_{2}\right| \geq k-n-1$. Let $X=X_{1} \cup\left\{\alpha_{1}, \alpha_{2}\right\}$ and $Y=E\left(M_{x y}^{p}\right)-X$. Then, $\min \{|X|,|Y|\}=n+1$. Therefore, By the similar argument in Theorem 4.7, one can easily show that $(X, Y)$ is a minimal $(n+1)$-separation for $M_{x y}^{P}$. 


\section{Applications}

In this section, we study the relations between the pinching operation and other binary operations such as splitting, the splitting off operation and element splitting. Using these relations, we will extend the notions of subdivision on to edges and special case of vertex identification on two vertices from graphs to binary matroids. Moreover, we shall show that Eulerian binary matroids are characterized in terms of the 2-pinching, splitting and splitting off operations.

The next theorem shows the relationship between $M$ and $M_{x y}^{p}$ by combining the notions of minors, the splitting off and element splitting operation.

Theorem 5.1. Let $M$ be a connected binary matroid. Let $x, y \in E(M)$ and $\Gamma=\left\{u, v, u^{\prime}, v^{\prime}\right\}$ such that $\Gamma \notin E(M)$. Consider the matroid $M_{x y}^{P}$ with $\delta=\left\{X_{1} \cup\left\{u, u^{\prime}\right\}, X_{2} \cup\left\{v, v^{\prime}\right\}\right\}$. Then

(i) $M_{x y}^{P} \backslash \Gamma=M \backslash\{x, y\}$.

(ii) $\left(\left(M_{x y}^{P}\right)_{u v}\right)_{u^{\prime} v^{\prime}} \cong\left(\left(M_{x y}^{P}\right)_{u^{\prime} v^{\prime}}\right)_{u v} \cong M$.

(iii) If $X_{1}=\emptyset$, then $M_{x y}^{P} /\left\{u, u^{\prime}\right\}=M_{x y}^{P} /\{u\} \backslash\left\{u^{\prime}\right\}=M_{x y}^{P} /\left\{u^{\prime}\right\} \backslash\{u\}=M$ where $\left\{u, u^{\prime}\right\}$ is a 2-circuit of $M_{x y}^{P}$. Moreover, $M_{x y}^{P}$ is a single parallel extension of $M_{x, y}^{\prime}$, or $M_{x y}^{P} \backslash u=M_{x, y}^{\prime}$ and $M_{x y}^{P} \backslash u^{\prime}=M_{x, y}^{\prime}$.

Proof. The proofs are straightforward.

A binary matroid $M$ with ground set $E$ is Eulerian if there exist disjoint circuits $C_{1}, C_{2}, \ldots, C_{\mathcal{P}}$ such that $E=C_{1} \cup C_{2} \cup \ldots \cup C_{\mathcal{P}}$ and $M$ is called bipartite matroid if every circuit has even cardinality. For a given connected graph $G$, the matroid $M(G)$ is Eulerian if and only if $G$ is an Eulerian [6]. A matroid $M$ is Eulerian if and only if $M_{x y}$ is Eulerian [3]. The next theorem, says that this property is preserved under 2-pinching operation. To prove this theorem, we consider the following lemma and we use the fact that $M$ is a binary matroid if and only if the symmetric difference of any set of circuits is a disjoint union of circuits [4].

Lemma 5.2. Let $M_{x y}^{P}$ be a pinching of a Eulerian binary matroid $M$. Then $E(M)$ can be partitioned into a collection of disjoint circuits of $M$ such that this partition contains $C_{x y}$.

Proof. For some $k$, Let $\pi=\left(C_{1}, C_{2}, \ldots, C_{k}\right)$ be a partition of $E(M)$ such that $C_{x y} \notin \pi$. Choose all circuits of this partition that have a non-empty intersection with $C_{x y}$. Let $V$ be the union of these circuits and let $V^{\prime}$ be the union of other circuits of this partition which are not in $V$. Then, there is a circuit $C$ that is not in $\pi$ and contains $x, y$ or both such that $C \cap V^{\prime}=\emptyset$ and $V \Delta C$ is a disjoint union of circuits and contains $C_{x y}$. Now $E(M)=V^{\prime} \cup(V \Delta C)$. Clearly, this union is a disjoint union of circuits.

Theorem 5.3. Let $M$ be a binary matroid on a set $E$ and $x, y \in E$. Then $M$ is Eulerian if and only if $M_{x y}^{P}$ is Eulerian.

Proof. Suppose that $M$ is a binary Eulerian matroid and $x, y \in E(M)$. Then, for some $k$, there are disjoint circuits $C_{1}, C_{2}, \ldots, C_{k}$ of $M$ such that $E(M)=\bigcup_{i=1}^{k} C_{i}$. Let $M_{x y}^{P}$ be a pinching of $M$. If $x \in C_{i}$ and $y \in C_{j}$ where $i, j \in\{1,2, \ldots, k\}$ and $i \neq j$, then $C^{\prime}=\left(C_{i}-\{x\}\right) \cup\left\{\alpha_{1}, \alpha_{2}\right\}$ and $C^{\prime \prime}=\left(C_{j}-\{y\}\right) \cup\left\{\alpha_{3}, \alpha_{4}\right\}$ are disjoint circuits of $M_{x y}^{P}$. Clearly, $E\left(M_{x y}^{P}\right)=C_{1} \cup \ldots \cup C_{i-1} \cup C^{\prime} \cup C_{i+1} \cup \ldots \cup C_{j-1} \cup C^{\prime \prime} \cup C_{j+1} \cup \ldots \cup C_{k}$ and these circuits are pairwise disjoint. If $x, y \in C_{i^{\prime}}$, for $i^{\prime} \in\{1,2, \ldots, k\}$, then we have two following cases.

(i) If $C_{x y}=C_{i^{\prime}}$ and $P=\left(X_{1}, X_{2}\right)$, then $E\left(M_{x y}^{P}\right)=C_{1} \cup \ldots \cup C_{i^{\prime}-1} \cup\left(X_{1} \cup\left\{\alpha_{1}, \alpha_{3}\right\}\right) \cup\left(X_{2} \cup\left\{\alpha_{2}, \alpha_{4}\right\}\right) \cup$ $C_{i^{\prime}+1} \cup \ldots \cup C_{k}$. Clearly, any two members of this union are disjoint. 
(ii) If $C_{x y} \neq C_{i^{\prime}}$, then we have two following cases:

-If $\left(\left(C_{i^{\prime}}-\{x, y\}\right) \cup\left\{\alpha_{1}, \alpha_{2}, \alpha_{3}, \alpha_{4}\right\}\right) \in \mathcal{C}_{3}$, then $E\left(M_{x y}^{P}\right)=C_{1} \cup \ldots \cup C_{i^{\prime}-1} \cup\left(C_{i^{\prime}}-\{x, y\}\right) \cup$ $\left.\left\{\alpha_{1}, \alpha_{2}, \alpha_{3}, \alpha_{4}\right\}\right) \cup C_{i^{\prime}+1} \cup \ldots \cup C_{k}$. Clearly, any two members of this union are disjoint.

-If $\left(\left(C_{i^{\prime}}-\{x, y\}\right) \cup\left\{\alpha_{1}, \alpha_{2}, \alpha_{3}, \alpha_{4}\right\}\right) \notin \mathcal{C}_{3}$, then by Lemma 5.2, there is another partition of $E(M)$ containing $C_{x y}$ and by part (i) of this proof we can see that $M_{x y}^{P}$ is an Eulerian matroid.

We conclude that $M_{x y}^{P}$ is an Eulerian matroid. To prove the converse, by Theorem 5.1, $M=$ $\left(\left(M_{x y}^{P}\right)_{\alpha_{1} \alpha_{2}}\right)_{\alpha_{3} \alpha_{4}}$. Therefore when $M_{x y}^{P}$ is an Eulerian matroid, $M$ is Eulerian.

Corollary 5.4. Every uniform matroid $U_{n, n+1}$ can be constructed from $U_{1,2}$ by a sequence of the 2pinching, splitting and splitting off operations.

Proof. Let $M=U_{1,2}$ and so there is no partition $P$. Then $M_{x y}^{\emptyset} \cong U_{1,2} \oplus U_{1,2}$. Now let $\left\{x^{\prime}, y^{\prime}\right\}$ be $\left\{\alpha_{1}, \alpha_{2}\right\}$ or $\left\{\alpha_{3}, \alpha_{4}\right\}$. Then $\left(M_{x y}^{\emptyset}\right)_{x^{\prime}, y^{\prime}} \cong U_{3,4}$ and $\left(\left(U_{3,4}\right)_{x^{\prime}, y^{\prime}}\right)_{x^{\prime} y^{\prime}} \cong U_{2,3}$. By repeating this process (first applying the 2-pinching operation with respect to partition $P$ with one empty part, then applying splitting and splitting off operations, respectively), we eventually obtain $U_{n, n+1}$ and then $U_{n-1, n}$, respectively.

On combining Theorem 5.3 with Corollary 5 , we immediately obtain the following result.

Theorem 5.5. A binary matroid is Eulerian if, by repeated applications of splitting and 2-pinching operations, it can be transformed to a direct sum of copies of $U_{1,2}$ and $U_{2,3}$.

Now we extend the notions of subdivision of edge and vertex identification from graphs to binary matroids by the pinching operation in the following way.

Let $G$ be a 2-connected graph and $x, y \in E(G)$. After applying the pinching operation on $x$ and $y$, let $\left\{x^{\prime}, y^{\prime}\right\}$ and $\left\{x^{\prime \prime}, y^{\prime \prime}\right\}$ be the disjoint subsets of members of $\delta$. Clearly, if we apply the splitting operation using $\left\{x^{\prime}, x^{\prime \prime}\right\}$ or $\left\{y^{\prime}, y^{\prime \prime}\right\}$, then the obtained graph is isomorphic to the graph resulting by subdivision of $G$ on $x$ and $y$.

Let $G$ be a 2-connected graph and $u, v, w, z \in E(G)$, such that $t_{1}$ and $t_{2}$ are the common vertices of $\{u, v\}$ and $\{w, z\}$, respectively, and there are no other edges on $t_{1}$ and $t_{2}$. Suppose that $\alpha$ and $\alpha^{\prime}$ are the new elements of the obtained graph after applying the splitting off operation on $\{u, v\}$ and then $\{w, z\}$, respectively. Now if we implement the pinching operation on $\left\{\alpha, \alpha^{\prime}\right\}$, then the obtained graph is isomorphic to the graph obtained by vertex identification of $t_{1}$ and $t_{2}$.

Definition 5.6. Let $M$ be a connected binary matroid and $x, y \in E(M)$. Consider the matroid $M_{x y}^{P}$ with $\delta=\left\{X_{1} \cup\left\{u, u^{\prime}\right\}, X_{2} \cup\left\{v, v^{\prime}\right\}\right\}$. We call $N_{1}$ as the subdivision of $M$ on $x$ and $y$ if $N_{1} \cong\left(M_{x y}^{P}\right)_{u, v} \cong$ $\left(M_{x y}^{P}\right)_{u^{\prime}, v^{\prime}}$.

Definition 5.7. Let $M$ be a connected binary matroid and $\Gamma \subseteq E(M)$ such that $\Gamma=\left\{u, v, u^{\prime}, v^{\prime}\right\}$. Suppose that $\{u, v\}$ and $\left\{u^{\prime}, v^{\prime}\right\}$ are cocircuits of $M$ and $E\left(M_{u v}\right)-E(M)=\{\alpha\}$ and $E\left(M_{u^{\prime} v^{\prime}}\right)-E(M)=\left\{\alpha^{\prime}\right\}$. We call $N_{2}$ is the identification $\{u, v\}$ with $\left\{u^{\prime}, v^{\prime}\right\}$ in $M$, if $N_{2} \cong\left(\left(M_{u v}\right)_{u^{\prime} v^{\prime}}\right)_{\alpha \alpha^{\prime}}^{P} \cong\left(\left(M_{u^{\prime} v^{\prime}}\right)_{u v}\right)_{\alpha \alpha^{\prime}}^{P}$.

Corollary 5.8. The matroids $N_{1}$ and $N_{2}$, defined as in definitions 5.6 and 5.7, are extensions of the notions of subdivision and vertex identification from graphs to connected binary matroids.

\section{References}

[1] G. Azadi, Generalized splitting operation for binary matroids and related results, Ph.D. Thesis, University of Pune, 2001. 
[2] A. Frank, Edge-connection of graphs, digraphs, and hypergraphs, More Sets, Graphs and Numbers 15 (2006) 93-141.

[3] M. M. Shikare, K. V. Dalvi, S. B. Dhotre, Splitting off operation for binary matroids and its applications, Graph and Combinatorics 27 (2011) 871-882.

[4] J. Oxley, Matroid Theory, Oxford University Press, 2nd ed. 2011.

[5] T. T. Raghunathan, M. M. Shikare, B. N. Waphare, Splitting in a binary matroid, Discrete Math. 184 (1998) 267-271.

[6] D. J. A. Welsh, Euler and bipartite matroids, Journal of Combinatorial Theory 6(4) (1969) 375-377.

[7] D. West, Introduction to graph theory, Prentice-Hall, 2nd ed. 2001. 